\title{
Philosophiques
}

\section{Le Québec et l'éthique libérale de la sécession}

\section{Guy Laforest}

Volume 19, numéro 2, automne 1992

Une nation peut-elle se donner la constitution de son choix?

URI : https://id.erudit.org/iderudit/027200ar

DOI : https://doi.org/10.7202/027200ar

Aller au sommaire du numéro

Éditeur(s)

Société de philosophie du Québec

ISSN

0316-2923 (imprimé)

1492-1391 (numérique)

Découvrir la revue

Citer ce document

Laforest, G. (1992). Le Québec et l'éthique libérale de la sécession.

Philosophiques, 19(2), 199-214. https://doi.org/10.7202/027200ar d'utilisation que vous pouvez consulter en ligne.

https://apropos.erudit.org/fr/usagers/politique-dutilisation/ 


\title{
LE QUÉBEC ET
}

\section{L'ÉTHIQUE LIBÉRALE DE LA SÉCESSION}

\author{
par \\ Guy Laforest
}

Il y avait quelque chose de prudent et de profondément sage dans la position adoptee par le Premier Ministre du Canada il y a cinquante ans, lors du tristement célèbre plébiscite sur la conscription. Loin de moi l'intention de vouloir soustraire monsieur Mackenzie King à la critique de son retour sur des engagements pris quelques années avant le plébiscite. Moralement, André Laurendeau et les militants du Bloc populaire avaient sans doute raison de lui en vouloir. Sauf que ramenée aux seules circonstances politiques de 1942, la position de Mackenzie King était fondamentalement saine: pas nécessairement la conscription, mais la conscription si nécessaire. Dans les conditions de la délibération politique, il n'était pas sûr que le gouvernement du Canada dût recourir à la conscription; l'évolution de la guerre aurait toutefois pu le forcer à le faire. En I942, Mackenzie King invita le Canada à respecter les paramètres de la morale aristotélicienne. Selon le penseur grec, les communautés politiques délibèrent toujours dans des circonstances qui leur sont particulières et qui peuvent changer.

$\mathrm{Vu}$ les dilemmes qui sont les siens en 1992, la communauté politique québécoise devrait suivre l'exemple de Mackenzie King et envoyer le message suivant au reste du Canada: pas nécessairement la sécession, mais la sécession si nécessaire. Je pense au fond que les orthodoxes de l'indépendantisme quëbécois et les inconditionnels du fédéralisme canadien méritent d'être rangés dans le même camp, celui d'un certain dogmatisme fondé sur 
une philosophie linéaire de l'histoire. Les premiers, comme Camille Laurin et Denis Monière, aiment bien le langage de la normalité. Il va de soi selon eux que les nations et les peuples, armés du sacro-saint droit à l'autodétermination, finiront par se transformer en États indépendants. Ils quitteront la dépendance de l'enfance, ou celle plus tourmentée de l'adolescence, pour parvenir à l'âge adulte et à la maturité. Il n'y a guère plus de doutes dans l'esprit des doctrinaires du fédéralisme comme Pierre Elliott Trudeau. Selon ce dernier, le sens de l'histoire est celui de l'intégration politique dans les grands ensembles. I'histoire du Canada - et celle du Québec, cela va de soi - sert d'exemple pour toute l'humanité. En 1977, devant le Congrès américain, monsieur Trudeau alla jusqu'à dire que l'indépendance du Québec serait un crime contre l'histoire de l'humanitè. La voie de la raison me semble ailleurs. L'indépendance du Québec n'est pas plus nécessaire sur le plan de l'histoire que criminelle sur celui de la morale.

Toute réflexion sur la problématique québécoise de la sécession doit s'appuyer sur nos circonstances particulières qui sont celles de l'enracinement canadien. Dans la première partie de ce texte, je proposerai un bilan de santé - en fait, un diagnostic de crise - du régime fédèral canadien en fin de vingtième siècle. Pour mieux situer le navire canadien sur la mer houleuse de la modernité tardive, je ferai appel à deux séries d'études qui viennent d'être rassemblées dans des mélanges offerts à ces deux piliers de la science politique au Canada et au Québec que sont Gérard Bergeron et Léon Dion. Dans un deuxieme temps, j'essayerai de monter une argumentation philosophique procurant des justifications à la sécession du Québec tout en respectant les paramètres de l'éthique libérale.

\section{Dans la ronde du Canada}

Pour bien comprendre la crise politico-constitutionnelle que nous traversons, il faut analyser la tourmente planétaire qui souffle sur la fin du vingtième siècle. La chute du mur de Berlin, l'effondrement du communisme et des empires multinationaux à l'Est, représentent autant de signaux d'une accélération de l'Histoire qui nous laisse encore abasourdis. Sur la mer agitée de la fin du siècle, il y a des mouvements et des forces qui se rencontrent, provoquant toutes sortes de bouleversements. D'un côté, on voit à l'oeuvre de puissantes tendances vers l'intégration globale: mondialisation des marchés, homogénéisation des sociétés grâce à l'expansion des technologies et des communica- 
tions, universalisation des preoccupations écologiques mais aussi généralisation des aspirations à une qualité de vie fondée sur la consommation. Jeffrey Simpson résume de la manière suivante la rationalité qui pousse vers une plus grande intégration économique:
Ainsi la globalisation, une expression qui évoque un mélange d'opportunités et de terreurs, signifie une explosion du commerce et de l'investissement, une ouverture accrue des grandes économies, une rationalisation industrielle à l'échelle mondiale, la création de contacts et d'alliances stratégiques entre les firmes et une diversifi- cation des centres mondiaux du pouvoir économique. Cette intégra- tion, ainsi que les exigences jumelées de la négociation de régles reconnues internationalement et de l'établissement de contrôles politiques sur les retombées de ces tendances, a obligé les pays a réflèchir a la question de savoir quelle portion de leur souveraineté serait cédée aux institutions supranationales ${ }^{\mathrm{I}}$.

Le Canada est inexorablement amené à s'engager davantage dans ce mouvement d'intégration économique. Après le libreéchange avec les Etats-Unis, nous connaîtrons le libre-échange à trois avec le Mexique, et cette entente sera peut-être généralisée à la grandeur des Amériques avant la fin du siècle. Dans ce contexte, pour le Canada mais aussi pour le Québec, le partage de la souveraineté est la voie de l'avenir. Mais si les sociétés et les peuples consentent à s'intégrer et à échanger de plus en plus les uns avec les autres, il ne sont pas pour autant disposés à renoncer à leur identité. Ils voudront conserver leur souveraineté sur les aspects les plus névralgiques de leur existence communautaire. La quête d'intégration économique peut s'accompagner d'un processus de fragmentation des États qui gèrent mal le pluralisme national et la diversité des peuples, chacun préférant négocier selon des priorites spécifiques son entrée dans la spirale de la globalisation. Au Québec, cette quête de sécurité identitaire s'est exprimée ces dernières années dans le débat autour de l'Accord du lac Meech: le Québec veut être reconnu en tant que société distincte, comme il veut avoir les moyens requis pour protéger et promouvoir ce qui fait son originalité en Amérique ${ }^{2}$. En résumé, nous nous demandons en 1992 comment le Québec pourrait rester lui-même en tant que société distincte et communauté

I. Jeffrey Simpson, "McLuhan, Frye and the Future of Canada 》, Queen's Quarterly, IC, 1, 1992, p. 13. (Traduction libre de Daniel Weinstock.)

2. Louis Sabourin vient de consacrer un bel essai au dilemme canadoquébécois dans la modernité tardive. Voir passion d'être désir d'avoir: Le dilemme Quebec-Canada dans un univers en mutation, Montreal, Boréal, 1992, p. 67 . 
nationale, sans que le Canada ne se sente encore plus vulnérable dans son propre projet identitaire, au moment où toutes les sociétés doivent affronter les dangers et les défis de la globalisation.

Les analystes et historiens qui se pencheront dans quelques années sur les paramètres politiques et idéologiques des délibérations canado-québécoises en Ig9r-Igg2 consulteront avec profit Être contemporain et L'engagement intellectuel, des mélanges honorant respectivement Gérard Bergeron et Léon Dion. Pionniers de la science politique au Québec, Bergeron et Dion ont consacré une partie importante de leur carrière et de leur œuvre à la question canado-québécoise. Il est heureux que l'on puisse retrouver dans des livres conçus pour les honorer des textes laissant entrevoir ce que l'avenir peut nous réserver. Je m’attarderai ici à des contributions d'intellectuels canadiens-anglais, auréolées d'un certain pessimisme, qui s'entendent pour conclure que le navire canadien a choisi un bien mauvais moment pour s'aventurer sur la mer dangereuse de la globalisation.

Le système politique canadien doit affronter les défis de l'époque contemporaine alors qu'il atteint des sommets inégalés de fragilité et de vulnérabilité. Dans une étude sur les mutations de la société canadienne-anglaise, John Meisel constate que le Canada n'est plus ce pays ouvert et tolérant, calme et civilisé, qu'il apprit à connaître puis à aimer en tant qu'immigrant originaire de la Tchécoslovaquie. L'échec de l'Accord du lac Meech a signalé la fin du régime de compromis et d'accommodements entre les élites francophones et anglophones sur lequel le fédéralisme canadien s'est appuyé pendant plus d'un siècle. Meisel note l'émergence de nouvelles couches d'élites, à la tête des groupes féministes, des organisations autochtones et multiculturelles. Ce phénomène est accompagné par un processus de fragmentation et de segmentation, dans là mesure où ces nouveaux dirigeants seront peu enclins à subordonner leurs intérêts sectoriels à quelque formulation du bien commun. Celles et ceux qui, comme John Meisel, Gérard Bergeron et Léon Dion, ont placé la dualité entre le Canada anglais et le Canada français - ou le Québec - au cœur de leur définition du pays, doivent maintenant déchanter:

Les valeurs des nouvelles élites diffèrent des valeurs traditionnelles, en ce qu'elles accordent peu (ou aucune) place à la définition dualiste de l'Etat canadien et au concept de l'unité nationale défini en termes de dualisme ... De sérieux désaccords d'une nature fondamentale, des conflits idéologiques de base, et une plus amère 
compétition pour les bénéfices ont altéré la substance ainsi que le style de la politique ${ }^{3}$.

Le système politique canadien est donc devenu plus conflictuel, plus difficile à gérer. L'adoption d'une Charte des droits et libertés, dans la Loi constitutionnelle de ig82, a renforcé l'individualisme et affaibli l'esprit de compromis. Désormais, des acteurs traditionnels jusqu'aux groupes les plus marginaux, on s'affirme et on se définit à partir des droits qui nous concernent et qui sont incorporés dans la Charte. Gare à ceux qui voudraient y toucher ou en modifier l'équilibre! Robert Bourassa et Brian Mulroney l'ont appris à leurs dépens pendant la saga du lac Meech.

Alors que la culture politique est en voie de redéfinition et de restructuration, le fédéralisme canadien voit certains de ses piliers vaciller. L'ombre de la fragmentation plane sur le système des partis, et celle de l'ingouvernabilité sur l'ensemble du gouvernement central. La montée de Preston Manning et du Reform Party dans l'ouest, celle de Lucien Bouchard et du Bloc québécois - chacun exprimant à sa façon le conflit des nationalismes dans le système -, risquent de donner des assises solides à cinq partis et ainsi de condamner le pays à une ère de gouvernements minoritaires. La crise du système des partis est accentuée par les multiples interrogations sur la qualite du leadership des partis traditionnels. Brian Mulroney, Jean Chrétien et Audrey Mclaughlin ne parviennent pas à convaincre les électeurs de leurs aptitudes à gouverner. La méfiance et le scepticisme règnent dans l'opinion publique. Et le vide dans les poches du gouvernement central. Pendant longtemps, Ottawa a pu se servir de ses largesses et de son pouvoir de dépenser pour cimenter ce pays diversifié, à la géographie impossible, qu'est le Canada. Cette époque paraît révolue. Avec un déficit qui dépasse les 400 milliards de dollars, le gouvernement fédéral n'aura bientôt plus les moyens de sa politique de redistribution et d'intervention dans les affaires provinciales, pourtant nécessaires à son effort « d'édification nationale ».

Lorsqu'un Etat central cesse d'avoir les moyens financiers de ses politiques centripètes, il peut être tenté de faire du

3. John Meisel, " Fin de siècle mutations in English Canada. Has the Peaceable Kingdom Turned Ugly », dans William Lapierre, Vincent Lemieux et Jacques Zylberberg, Être contemporain. Melanges en lhonneur de Gérard Bergeron, Sillery, Presses de l'Université du Québec, I992, p. 235. (Traduction libre de Daniel Weinstock.) 
surinvestissement du côté des normes et des symboles. Il pourrait essayer de resserrer davantage les mailles de la vision du pays que Pierre Elliott Trudeau a fait triompher, celle de l'Étatnation bilingue et multiculturel. Mais comme le regretté Donald Smiley et Blair Neatby l'on fait remarquer respectivement dans les mélanges offerts à Bergeron et Dion, le bilinguisme et le multiculturalisme ont cessé d'être - s'ils l'ont jamais èté - des symboles unificateurs. Smiley écrit ce qui suit à propos du bilinguisme
La séparation territoriale persistante des deux groupes linguistiques signifie que dans leur quotidien la plupart des citoyens de l'un ne sont pas en contact avec des membres de l'autre. Ainsi, le fait de soutenir la dualité linguistique comme caracteristique primordiale du Canada est, au mieux, artificiel dans des endroits tels que Victo- ria, Brampton ou Corner Brook, et au pire, perçu comme l'imposition d'un régime presqu'entièrement étranger aux intérêts et aux traditions de ces endroits ${ }^{4}$.

Dans le même texte, Smiley écrit qu'il considère absurde le traitement symétrique accordé aux communautés linguistiques minoritaires, tant francophones qu'anglophones, dans les politiques de l'État central ${ }^{5}$. Quant à Blair Neatby, il voit poindre une contradiction entre la politique officielle de dualité linguistique et la reconnaissance octroyée au multiculturalisme en tant que caractéristique fondamentale du Canada ${ }^{6}$.

La crise politique canadienne, outre ses dimensions institutionnelle et structurelle, prend donc aussi des connotations symboliques. Il $y$ a mésentente sur les normes de base qui devraient servir de piliers au vouloir-vivre collectif. Au moment où monte aussi l'aliénation et les revendications des peuples autochtones, les visions dominantes au Canada anglais - la nation multiculturelle d'un Pierre Elliott Trudeau -, et au Québec - l'État fédèral et binational d'un André Laurendeau -, semblent incapables d'intégrer la complexité du reel canadien dans une nouvelle synthèse. Telle est la conclusion à laquelle en arrive Michael Oliver, l'ex-directeur de la recherche de la Commission Laurendeau-Dunton sur le bilinguisme et le biculturalisme:

4. Donald Smiley, " Language Policies in the Canadian Political Community », dans Lapierre, Lemieux et Zylberberg, Op. cit., p. 287. (Traduction libre de Daniel Weinstock.)

5. Ibid., p. 284.

6. H. Blair Neatby, « Le mécontentement de l'après-Meech », dans Raymond Hudon et Réjean Pelletier, L'engagement de lintellectuel. Melanges en l'honneur de Léon Dion, Sainte-Foy, Presses de l'Université Laval, 1991, p. 381. 
Une des façons d'affronter les erreurs des théories du nationalisme et du fédèralisme que nous avons ètudiées, c'est de les considèrer du point de vue de la communauté. Pour Laurendeau, c'est la société nationale qui est une communauté privilègiée. Pour Trudeau, cest l'Etat multiculturel. Aucun ne semble fournir le cadre de référence qui nous permettrait de réflèchir de manière constructive aux problèmes des années quatre-vingt-dix ${ }^{7}$.

Encore mal à l'aise dans une nouvelle culture politique plus conflictuelle, aux prises avec une crise de légitimité du leadership fédéral et l'amorce d'une fragmentation de son système de partis, alourdi par une dette qui le rend de moins en moins capable de poursuivre un travail d'édification « nationale », et freiné de toute façon sur cette voie par des malentendus à propos du sens de certains symboles fondamentaux, l'État central canadien paraît bien mal en point. Ballotté sur les eaux tumultueuses de la globalisation, confronté à une foule de problèmes internes, le navire canadien doit trouver en même temps le moyen de se refaire une constitution.

L'Accord du Lac Meech, c'était la ronde du Québec. Comme le gouvernement et l' Assemblée nationale du Québec n'avaient pas signé la Loi constitutionnelle de I982, on allait trouver une façon de remédier à cela et de procurer des garanties au Québec, notamment en le reconnaissant à titre de société distincte. Sans vouloir simplifier outrageusement, je suggèrerais que les signataires du pacte Meech en 1987 avaient oublié que le Québec n'est pas le seul à souffrir d'angoisses identitaires en Amérique, en fin de vingtième siècle. Déjà échaudès par un affaiblissement de l'Etat central par l'entremise du traité de libre-échange avec les Etats-Unis, les nationalistes canadiens sursautèrent devant les concessions faites par Ottawa au lac Meech, celles qui furent accordées uniquement au Québec comme celles qui furent aussi octroyées aux autres provinces. Pour ces gens, le principe d'une nouvelle ronde de négociations constitutionnelles ne deviendrait acceptable que si les discussions servaient à remodeler et renforcer le Canada lui-même. Entre ig87 et 1992, nous sommes donc passés de la ronde du Québec à celle du Canada. Les principales étapes de la ronde du Canada ont été jusqu'à présent les suivantes?: un Forum des citoyens, présidé par Keith Spicer, s'est fait dire par les « Canadiens ordinaires » que les compromis de demain « ne pourraient intervenir que dans le cadre d'un pancanadianisme fort oủ tous les Canadiens jouiraient de droits

7. Michael Oliver, "Laurendeau et Trudeau: leurs opinions sur le Canada », dans Hudon et Pelletier, Op.cil., p. 362. 
égaux, de normes nationales et d'un même accès aux programmes et services ${ }^{8}$. À la même époque, à l'hiver I99r, Gérald Beaudoin et Jim Edwards ont dirigé les travaux d'un comité du Sénat et de la Chambre des Communes sur la formule d'amendement de la constitution, recommandant l'adoption d'un mécanisme de veto régionaux. En septembre iggi, dans un document intitulé Bâtir ensemble l'avenir du Canada, le gouvernement fédéral soumit à l'ensemble des intèressés une série cornplexe de propositions visant à renouveler le fédéralisme. Pour accompagner le processus de réflexion, Ottawa mit aussi sur pied un autre Comité du Sénat et de la Chambre des Communes, sous la responsabilité de Claude Castonguay (vite remplacé par Gérald Beaudoin) et Dorothy Dobbie. Après des débuts pénibles, et un effort de redressement qui culmina dans une série de cinq conférences réunissant des responsables gouvernementaux, des experts, des représentants des groupes d'intérêts et des citoyens ordinaires, le Comité Beaudoin-Dobbie publia son rapport, Un Canada renouvelé, le 29 février Ig92. La ronde du Canada continua de plus belle avec une autre série de réunions multilatérales sur la constitution, de mars à juin 1992, avec la participation du gouvernement fédéral, des gouvernements de toutes les provinces sauf le Québec, des leaders des quatre organisations autochtones et des deux territoires. Après toutes ces palabres, l'impasse continue de persister.

Compte tenu de l'ampleur des réformes envisagées, il n'est pas vraiment surprenant de constater l'accumulation des nuages au-dessus de la ronde du Canada. Les parties essaient de s'entendre sur un nouveau partage des pouvoirs, rompant ainsi avec un ordre en vigueur depuis 125 ans. On souhaite aussi réformer des institutions centrales comme la Cour suprême et le Sénat. Pour ce qui est de la deuxième chambre législative, certains gouvernements aspirent même à une véritable révolution; ils veulent en effet doter le Sénat de nouveaux pouvoirs, faire élire ses membres et octroyer une représentation égale à toutes les provinces. En plus de régler une fois pour toutes la question du Québec et celle de l'intégration des peuples autochtones, toutes deux symboliquement fort chargées, les participants à la ronde du Canada doivent aussi définir les paramètres d'une charte sociale et d'une nouvelle union économique. À une époque où, comme nous

8. Le Forum des citoyens sur l'avenir du Canada, Rapport à la population et au gouvernement $d u$ Canada, Ottawa, Ministère des approvisionnements et services du Canada, 1991, p. 66. 
l'avons vu, le système politique canadien paraît particulièrement faible et vulnérable, l'ambition réformatrice des acteurs perd tout sens de la mesure. C'est dans un tel contexte, celui d'un régime fédéral canadien qui donne des signes d'engagement dans un processus de lente désintegration, que toute réflexion sur une démarche sécessionniste québécoise mérite d'être replacée.

\section{I'ÉTHIQUE LIBÉRALE DE LA SÉCESSION}

Vers la fin de 1988 ou au début de 1989 , l'historien britannique E.J. Hobsbawm écrivit les phrases suivantes, qu'il vaut la peine de méditer:

Après tout, le fait mème que les historiens aient au moins commencé à progresser dans l'étude et l'analyse des nations et du nationalisme suggère, comme c'est souvent le cas, que le phénomene a passé son apogée. La chouette de Minerve qui apporte la sagesse, disait Hegel, ne vole qu'au crépuscule. C'est un bon signe de constater qu'elle est maintenant en train de tourner autour des nations et du nationalisme ${ }^{9}$.

La chouette de Minerve avait bel et bien pris son envol au crépuscule de la dernière décennie, Hobsbawm ne s'y est pas trompé. Sauf qu'elle n'irait pas là ou il l'avait prévu. Le vol de la chouette n'annonçait pas la mort du nationalisme, mais plutôt celle du communisme de même que le déclin des structures fédéralistes un tant soit peu artificielles. Les fédérations soviétique et yougoslave ne sont plus, tandis que la Tchécoslovaquie parait moribonde. A tour de rôle la Lituanie, la Lettonie, l'Estonie, l'Ukraine, la Slovénie, la Croatie, et d'autres encore ont fait sécession, dans une remarquable résurgence du nationalisme en fin de vingtième siècle. Tout aussi hors du commun fut le flair du philosophe américain Allen Buchanan, qui publia en Iggi un livie dont le titre révèle la pertinence indubitable: Secession. The Morality of Political Divorce from Fort Sumter to Lithuania and Quebec. En m'appuyant sur le travail de Buchanan, tout en le critiquant à certains égards, je vais maintenant essayer de voir comment se pose le problème de la sécession du Québec, dans l'oeil de l'éthique libérale.

Le livre de Buchanan n'envisage pas de régler de façon a priori toutes les difficultés entourant la réflexion lucide et critique, à propos de la sécession. L'auteur admet d'emblée qu'il n'y a pas de

9. E. J. Hobsbaw, Nations and Nationalism since 1780 . Programme, Myth, Reality, Cambridge, Cambridge University Press, 1989, p. 183. (Traduction libre de Daniel Weinstock.) 
théorie morale dans l'absolu, que son discours philosophique n'élimine pas l'urgence de la délibération politique pour les gens qui, sur le terrain, ont à considérer les dilemmes de la sécession. Selon Buchanan, ma reformulation du message de Mackenzie King est valable tant pour la théorie que pour la réalité empirique des sociétés visées: pas nécessairement la sécession mais la sécession si nécessaire. On commence à mieux saisir la portée de l'analyse de Buchanan pour le Québec lorsque l'auteur écrit que la protection des droits individuels n'est pas la seule raison que l'on peut invoquer pour faire sécession. Selon lui, semblable rupture peut être justifièe également lorsque des gens veulent promouvoir une autre conception de la communauté que celle qui prévaut dans leur régime politique, ou encore lorsqu'ils veulent protéger une culture originale ${ }^{10}$. L'auteur va jusqu'à affirmer qu'il $n^{\prime} y$ a rien qui empêche que les droits des groupes, les droits collectifs, puissent coexister avec les droits individuels dans la philosophie libérale. Il pense que le droit à la sécession représente un de ces droits qui ne sauraient être exercés que par une communauté dans son ensemble, au nom d'une collectivité ${ }^{\mathrm{II}}$.

Il n'y a rien dans le livre de Buchanan qui puisse s'apparenter à un plaidoyer en faveur de la sécession du Québec. Bien au contraire. D'abord, bien qu'il y soit beaucoup question du Canada et du Québec, le livre sappuie sur des connaissances historiques assez limitées. S'il rappelle correctement la Conquête de i763 et disserte assez longuement sur l'Accord du lac Meech, Buchanan ne se penche pas sur le compromis de i867. Il omet par ailleurs de mentionner que la Charte canadienne des droits et libertés, document-clè de la révision constitutionnelle de rg82, fut enchâssée sans le consentement des autorités québécoises. Quant aux indépendantistes ils sursauteront en apprenant que le Québec, d'après Buchanan, n'a pas encore épuisé toutes les options qui s'offrent à lui dans un régime fédéral, en deça de la sécession $^{12}$. Le philosophe américain pense que le Québec pourrait conserver une autonomie politique suffisante grâce à des mécanismes comme une clause dérogatoire semblable à la clause nonobstant que l'on retrouve dans la constitution de ig82, grâce aussi à un droit de veto sur les amendements constitutionnels. À l'heure où ces lignes sont écrites, incidemment, il convient de

10. Allen Buchanan, Secession. The Morality of Political Divorce from Fort Sumter to Lithuania and Quebec, Boulder, Colorado, Westview Press, I99ı, Pp. 6-7.

II. Ibid., P. 74.

12. Ibid., P. I6 
rappeler que cette question d'un droit de veto pour le Québec s'avère l'un des principaux obstacles à la résolution de la crise constitutionnelle.

Quand la culture politique d'un pays est de plus en plus dominee par le langage des droits individuels, il y a aussi un prix à payer pour l'utilisation d'une clause dérogatoire. Voici ce que Alain Baccigalupo vient d'écrire à ce sujet:

A l'intérieur d'une société démocratique qui porte haut l'étendard
du libéralisme et qui s'avère très soucieuse de protéger et de défen-
dre ses libertés fondamentales, il va de soi que ceux qui sont élus
par les suffrages de leurs commettants, selon les mécanismes que
l'on connaît ne sont en général pas très à l'aise - c'est le moins que
l'on puisse dire - avec l'idèe de devoir recourir fréquemment à la
clause dérogatoire.

Les conséquences politiques de tels agissements non seulement ne se feraient pas attendre mais pourraient bien s'averer, en pratique, beaucoup plus graves que les effets juridiques auxquels la puissance publique aurait voulu èchapper. ${ }^{13}$.

Si Buchanan désirait des précisions à ce sujet, il n'aurait qu'à les demander à Robert Bourassa, qui devra vraisemblablement recourir à la clause nonobstant en décembre ig93 pour continuer d'appliquer les articles de la Loi 178 relatifs à la langue d'affichage. Le philosophe américain me semble en terrain plus solide lorsqu'il écrit que le droit des peuples à l'autodétermination, figure de proue de la rhétorique nationaliste, ne représente pas un motif particulièrement fort en faveur de la sécession. Il estime en effet que ce droit est trop vague et que sa généralisation conduirait à une fragmentation politique illimitée ${ }^{\mathrm{I4}}$. Plusieurs des arguments en faveur de la sécession analysés par Buchanan me semblent au demeurant d'une pertinence toute relative dans la situation canado-québécoise. On ne se lancera pas ici dans l'aventure de la sécession pour augmenter de façon marginale l'efficacité de l'économie ou encore pour échapper à des relents de discrimination dans les politiques distributives.

Pour qu'une démarche sécessionniste soit légitime selon les paramètres de la pensée libérale, Buchanan estime que ceux qui l'entreprennent doivent pouvoir recourir à une pluralité de facteurs, qui se renforcent et se complètent les uns les autres. En

13. Alain Baccigalupo, « Le système politique canadien depuis l'avènement de la charte: démocratie ou juriscratie? » dans Louis Balthazar, Guy Laforest et Vincent Lemieux, Le Québec et la restructuration du Canada 1980-1992, Sillery, Les Editions du Septentrion, 1992, p. 131.

14. Buchanan, Op.cit., p. 49. 
dépit des nuances que je viens de faire, son livre permet de dégager l'allure générale d'une argumentation semblable dans le cas du Québec. Trois facteurs s'unissent pour former selon moi la combinaison gagnante pour le Québec de I9g2: la révision de la constitution canadienne en ig82 sans le consentement du Québec; le besoin de donner aux institutions du Québec les instruments nécessaires à la protection et à la promotion d'une culture et d'une société distinctes en Amérique; l'approfondissement de la diversité au sein de la société québécoise.

Buchanan opère une distinction fondamentale entre le droit à la révolution et le droit à la sécession. Alors que les sécessionnistes veulent soustraire un territoire et des citoyens de l'autorité d'un certain gouvernement, les révolutionnaires, eux, veulent renverser un gouvernement pour faire des changements constitutionnels, économiques ou socio-politiques à l'intérieur dun Etat ${ }^{15}$. L'auteur rappelle que pour le philosophe libéral John Locke le droit à la révolution comme le droit de former une association politique, était un droit collectif que l'on pouvait exercer lorsqu'un régime perd sa légitimité en violant les droits individuels fondamentaux ${ }^{16}$. Buchanan èlargit la pensée de Locke sur le droit de résistance aux régimes fédéraux:

Afin de s'adapter aux systèmes fédéraux, nous pouvons ajouter aux
droits individuels libèraux traditionnels des droits d'Etats constitu-
tionnellement désignés (afin de préciser une division du travail
entre les fonctions fédérales et celles des Etats). Cet ajout donne lieu
a une conception èlargie de l'injustice gouvernementale et à une
conception ètendue correspondante des conditions justifiant la
révolution: si le gouvernement central viole les droits individuels de
base ou les droits des Etats, on peut lui résister avec force ${ }^{17}$.

J'estime que les Québécois jouissent d'un tel droit par rapport aux institutions fédérales canadiennes. En 1982, la constitution canadienne a été rapatriée et amendée, - et la division du travail entre entités fédérées et gouvernement central a été changeee, selon les termes de Buchanan -, sans le consentement du gouvernement du Québec, sans celui de l'Assemblée nationale, et sans que la population du Québec ait èté antérieurement ou ultérieurement consultée de façon directe sur cette question, soit lors d'une élection fédérale ou d'une campagne référendaire. Qui plus est, la réforme fut effectuée moins de deux ans après

15. Ibid., p. Io.

16. Ibid., p. 77. Locke comme on le verra dans un moment, allait plus loin que cela.

17. Ibid., p. 38. (Traduction libre de Daniel Weinstock.) 
des promesses notoirement ambiguës de renouvellement du fédéralisme, faites aux Québécois par monsieur Trudeau lors du référendum de mai 1980 . Pour empirer encore la situation, les articles sur les droits linguistiques et scolaires qui sont le coeur même de la Loi constitutionnelle de 1982 et de la Charte des droits (particulièrement l'article 23) heurtaient de front et diminuaient les pouvoirs législatifs de l'Assemblée nationale du Québec encore une fois sans son consentement. Cette attaque de l'autonomie législative du Québec n'était pas accidentelle. Considèrons à ce propos l'analyse de Kenneth McRoberts:

Deuxième volet de la stratégie de Trudeau: le renforcement des droits linguistiques. Son moyen: une charte des droits enchâssée dans la Constitution. La Charte des droits traite d'une multitude de sujets autres que les droits linguistiques. Mais ces droits linguistiques sont incontestablement sa raison d'être ...

Les stratèges fédèraux espéraient aussi que les Québécois, à l'instar des Canadiens anglais verraient dans la Charte une déclaration de leurs droits et que, par conséquent, ils se sentiraient plus attachès à la communauté politique canadienne ${ }^{18}$.

Je pense que tout cela rend la Charte des droits et libertés profondement illégitime, et injuste, au Québec. Non seulement ce document a-t-il été imposé aux autorités et à la population du Québec sans leur consentement, mais en plus il s'en prend à des pouvoirs considérés essentiels! De mon point de vue, depuis le I7 avril I982, le pacte de confiance entre le gouvernement fédéral et le Québec est brisé. Je reconnais, avec Buchanan, que le droit à la révolution, le droit de se doter d'un nouveau régime et d'un nouveau gouvernement ne permet pas de conclure nécessairement à un droit de sécession. Il faut aussi que les titulaires du droit à la révolution puissent prétendre à la possession et au contrôle du territoire en question ${ }^{19}$. Je ne voudrais pas me lancer ici dans les méandres du droit international. Du point de vue de l'histoire et de la pensée politique, je me sens assez proche de la thèse de Buchanan:

La prètention à l'effet que le Canada anglais, qui a annexé le Quẻbec a l'occasion d'une guerre impériale, n'a pas de droit à l'égard de ce territoire, est beaucoup plus plausible que celle selon laquelle les Québécois auraient droit au territoire dans sa totalitée ${ }^{20}$.

L'absence de consentement du Québec laisse planer des doutes très importants quant à la légitimité de la réforme de

I8. Kenneth McRoberts, « Dans l'oeil du castor », Possibles, XVI, 2, I992, p. 40. 19. Buchanan, Op.cil., p. 72.

20. Ibid., p. n1. (Traduction libre de Michel Seymour.) 
1982 et des institutions fédérales au Québec. Sans être une condition suffisante, cela représente néanmoins une carte très importante dans l'argumentation pro-sécessionniste. Je vois dans le besoin de protéger l'intégrité d'une culture le deuxième motif amenant le Québec à vouloir quitter la fédération. En théorie, un système fédéral est tout à fait compatible avec l'objectif de protection et de promotion de la culture québécoise. En pratique, le Quëbec a toutes les difficultés du monde à obtenir ou conserver les outils législatifs et institutionnels lui permettant de réaliser cet objectif. Comme on l'a vu, le Canada s'est doté en I982 d'une Charte des droits qui visait intentionnellement les lois linguistiques québécoises. En Iggo l'Accord du lac Meech, qui donnait au gouvernement et à l'Assemblée nationale des obligations de protection et de promotion d'une société distincte au Québec, a èté rejeté. Même si la Charte contient un equilibre entre droits individuels et collectifs, le rejet de la clause de la société distincte signifie que dans la culture politique canadienne-anglaise, le libéralisme monochrome et symétrique, celui que promeuvent des gens comme David Bercuson et Barry Cooper, est en train de s'imposer. Charles Taylor interprète de la façon suivante le dérapage de l'Accord du lac Meech:

Et le Québec a vu que le geste cherchant à donner préséance à la Charte imposait une forme de société libérale étrangère, à laquelle le Québec ne pourrait jamais s'adapter sans abandonner son identité II $^{2}$.

Pour que la protection culturelle puisse servir de justification à une entreprise sécessionniste, Buchanan croit que cinq conditions doivent être remplies ${ }^{22}$. Je les reprends en ordre inverse de leur présentation. D'abord, le statut du territoire doit être clair. Sur cette question, vu l'ambiguité du droit international et de la pensée politique, le Québec ne pourra pas faire l'économie de négociations politiques avec les peuples autochtones. Il faut aussi que l'indépendance ne mène pas à l'édification d'un Etat qui bafoue les principes libéraux, sur la base d'une culture qui ne respecterait pas les normes fondamentales de justice. D’après Buchanan, le Québec est en terrain solide à propos de ces deux conditions. Il faut aussi que l'on ait épuisé les mécanismes normaux à l'intérieur du régime dont on veut faire sécession.

2I. Charles Taylor, "Shared and Divergent Values » dans Ronald Watts et Douglas M. Brown, Options for a New Canada, Toronto, University of Toronto Press, I991, p. 71. (Traduction libre de Daniel Weinstock.)

22. Buchanan, Op.cil., p. 6 . 
Pendant combien de décennies la société québécoise devra-t-elle consacrer le meilleur d'elle-même au renouvellement du fédéralisme pour satisfaire cette condition? Enfin, il faut que la culture en question soit vraiment en peril. Une telle situation de fragilité peut se démontrer par toutes sortes d'indicateurs empiriques. Doune certaine façon, la langue française et la culture québécoise seront toujours vulnérables en Amérique du Nord. Jen resterai dans ce texte à la dimension idéologique de l'affaire. Au Canada anglais, dans la Charte et dans l'opinion publique, il y a plusieurs idéologies qui saffrontent, plusieurs conceptions de l'État et de la citoyenneté. Selon Andrew Stark elles se rejoignent toutes dans leur refus d'admettre de façon symbolique et substantielle la spécificité de la communauté québécoise:

Chacune de ces conceptions fonctionne, respectivement, pour transcender, contrecarrer ou diminuer le caractère distinct du Québec à l'intérieur du Canada ${ }^{23}$.

Le projet d'édification d'une seule et unique nation canadienne met en péril le projet de promotion d'une identité et d'une culture distinctes au Québec. La Charte oeuvre à déterritorialiser les identités « locales » et « régionales » au profit de la communauté nationale canadienne et des institutions centrales. Si j'ai raison dans mon interpretation, et si le Canada s'apprête à s'effondrer du fait de son incapacité à reconnaitre le pluralisme national, le Québec devrait en tirer une leçon capitale.

Si le Québec se lançait sur la voie de la sécession pour imposer à tous ses citoyens un même ensemble de valeurs fondamentales - selon la logique de monsieur Trudeau - ou encore pour faire triompher des conceptions «monochromes » de l'identité et de l'appartenance communautaires, il referait les mêmes erreurs que ce Canada qui a substitué un projet nationaliste au fédéralisme complexe en 1982. Outre les motifs déjà invoqués, le Québec devrait se réclamer de l'approfondissement de la diversité dans sa rhétorique sécessionniste. La société distincte québécoise devrait se montrer plus forte et plus généreuse que le Canada de 1982 et de la Charte des droits et libertés dans son acceptation de la diversité authentique, en reconnaissant le pluralisme national sur son territoire. La constitution du Québec et les institutions parlementaires devraient reflèter ce pluralisme, l'existence de communautés nationales francophones, anglophones et autochtones.

23. $\Lambda$ Stark, "English Opposition to Quebec Nationalism » dans Kent Weaver (dir.), The Collapse of Canada?, Washington D.C., Brookings Institution, 1992, p. 157. 
Les méthodes de la science politique, telles que je les applique, laissent entrevoir que le Québec risque de continuer de tourner en rond dans un Canada paralysé par la multiplicité des problèmes qui l'assaillent. Les paramètres de la pensée libérale, tels que je les interprète, autorisent la communauté québécoise, en théorie tout au moins, à faire sécession de la fédération canadienne. Les Québécois vont-ils, ou devraient-ils, marcher sur ce terrain? En définitive, c'est à eux qu'il reviendra de le dire, et non à la science politique et à la philosophie.

Département de sciences politiques

Université Laval 